VOL. 9 (1973), 267-274.

\title{
Finite groups which are the product of two nilpotent subgroups
}

\section{Fletcher Gross}

\begin{abstract}
Suppose $G=A B$ where $G$ is a finite group and $A$ and $B$ are nilpotent subgroups. It is proved that the derived length of $G$ modulo its Frattini subgroup is at most the sum of the classes of $A$ and $B$. An upper bound for the derived length of $G$ in terms of the derived lengths of $A$ and $B$ also is obtained.
\end{abstract}

\section{Introduction}

Suppose $G$ is a finite group which is the product of two nilpotent subgroups $A$ and $B$. That $G$ must be solvable was proved by Kegel [5]. It has been conjectured that $d(G)$, the derived length of $G$, is at most $C(A)+C(B)$ where $C(A)$ and $C(B)$ denote the class of $A$ and $B$, respectively. This conjecture has been verified only in two special cases:

(1) when $A$ and $B$ are both abelian (Itô [4]), and

(2) when $A$ and $B$ have relatively prime orders (Hall and Higman [3]).

One of the principal theorems of the present paper is that if $D(G)$ is the Frattini subgroup of $G$, then $d(G / D(G)) \leq c(A)+c(B)$. As a result, the problem of finding some upper bound for $d(G)$ in terms of $C(A)$ and $c(B)$ is reduced to finding a bound on $d(D(G))$. But $D(G)$ is nilpotent, and, if $P$ and $Q$ are Sylow $p$-subgroups of $A$ and $B$, respectively, $a$ theorem of Wielandt [6] implies that $P Q$ is a Sylow p-subgroup of $G$. Hence, if $f(x, y)$ were some function such that whenever $S=P Q$, where

Received 8 May 1973. The research was supported in part by a grant from the National Science Foundation of the USA. 
$S$ is a $p$-group and $P$ and $Q$ are subgroups, it followed that $d(A) \leq f(c(P), c(Q))$, then it would be true that $d(G) \leq f(c(A), c(B))+c(A)+c(B)$. Thus one consequence of this paper is that the original problem is reduced to a problem concerning p-groups. Unfortunately, $G / D(G)$ is abelian if $G$ is a p-group, and so the results of this paper are trivial for p-groups.

It is also possible to bound $d(G / D(G))$ in terms of other invariants of $A$ and $B$. For example, if the Sylow 2-subgroups of both $A$ and $B$ have class at most 2 , then $d(G / D(G)) \leq d(A) d(B)+1$. Under a similar hypothesis, the nilpotent length of $G$ is at most $d(A)+d(B)$. In comparing these results with the previous one, it should be remembered that, in general, $d(A)$ is much smaller than $c(A)$ (to be more specific, $\left.d(A) \leq 1+\log _{2}(c(A))\right)$. I conjecture that the requirement on the Sylow 2-subgroups of $A$ and $B$ in these theorems is unnecessary.

\section{Preliminaries}

All groups considered in this paper are finite and solvable. $F(G)$ and $D(G)$. denote the Fitting and Frattini subgroups of $G$, respectively. $G^{\prime}$ is the derived group of $G$ and $G^{(n)}$ is defined inductively by $G^{(0)}=G$ and $G^{(n+1)}=\left(G^{(n)}\right)^{\prime} \cdot d(G)$ and $Z(G)$ are the derived length and nilpotent length, respectively, of $G$. If $G$ is nilpotent, $c(G)$ is the class of $G$. If $p$ is a prime, then $Z_{p}(G)$ is the $p$-length of $G$ and $O_{p}(G)$ and $O_{p^{\prime}}(G)$ are the largest normal p-subgroup and $p^{\prime}$-subgroup of $G$, respectively. $O_{p p^{\prime}}(G)$ is defined by $O_{p}\left(G / O_{p}(G)\right)=O_{p p^{\prime}}(G) / O_{p}(G)$. If $A$ and $B$ are subsets of $G$, then $[A, B]$ is the subgroup of $G$ generated by all elements of the form $x^{-1} y^{-1}$ xy where $x \in A$ and $y \in B$. If $C$ is a third subset, then $[A, B, C]=[[A, B], C], C_{G}(A)$ is the centralizer of $A$ in $G$ and $Z(G)$ is the center of $G .|S|$ denotes the number of elements in the set $S$.

If $n$ is a positive integer, $F_{n}$ denotes the collection of all groups $G$ satisfying $d(G / D(G)) \leq n$. It is an easy exercise to verify 
that the group $G$ belongs to $F_{n}$ if, and only if, $G^{(n-1)}$ is nilpotent. It is immediate from this that $F_{n}$ is a saturated formation in the sense of Gaschütz [1]. $N_{n}$ is the collection of all groups $G$ of nilpotent length at most $n \cdot N_{n}$ is also a saturated formation.

LEMMA 1. Suppose $G$ is a group and $F$ is a saturated formation such that $G \notin F$ but $F$ contains every proper homomorphic image of $G$. Then $D(G)=1$ and $G$ has only one minimal normal subgroup.

This follows directly from the definition of a saturated formation.

LEMMA 2. Let $G$ be a group, $p$ a prime, and $P$ a Sylow p-subgroup of $G$. Assume that $o_{p}(G)=1$ and that either $p>2$ or $c(P) \leq 2$. Then $d\left(P / O_{p}(G)\right) \leq d(P)-1$.

This follows from [3, Theorem 3.2.1] if $p>3$, from [3, Theorem 3.2.2] if $p=3$, and from [3, Lemme 1.2.3] if $p=2$.

LEMMA 3. Let $P$ be a Sy low 2-subgroup of $G$ and assume that $c(P)=3$. Then $d(P)=2$ and $z_{2}(G) \leq 2$.

Proof. Without loss of generality, we may assume that $O_{2},(G)=1$. $P$ is not abelian and $P^{(2)} \subseteq[P, P, P, P]=1$. Hence $d(P)=2$. Let $V$ be $O_{2}(G) / D\left(O_{2}(G)\right)$ written additively. Since $[y, x, x, x]=1$ for all $x$ and $y$ in $P$, it follows that if we represent $G / O_{2}(G)$ as a linear group operating on $V$, then $G / O_{2}(G)$ satisfies the hypothesis of Theorem 3.1 of [2]. Hence $\tau_{2}\left(G / O_{2}(G)\right) \leq 1$ by that theorem. Lemma 3 now follows.

THEOREM 1. Assume that $A$ and $B$ are proper nilpotent subgroups of the group $G$ such that $G=A B$. Assume that $D(G)=1$ and that $G$ has only one minimal normal subgroup $M$. Let $p$ be a prime dividing $|M|$. Then $M=O_{p}(G)=F(G) \neq G$ and one of $A$ and $B$ is a sylow p-subgroup of $G$ while the other is a Hall $p^{\prime}$-subgroup of $G$.

Proof. Since $G$ is solvable, $M$ is an elementary abelian p-group. Due to the uniqueness of $M, F(G)=O_{p}(G) \supseteq M$. Let $J$ and $K$ be the 
Hall $P^{\prime}$-subgroups of $A$ and $B$, respectively. Let $P$ and $Q$ be the Sylow $p$-subgroups of $A$ and $B$, respectively. Then by a theorem of Wielandt [6], $P Q$ is a Sylow $p$-subgroup of $G$ and $J K$ is a Hall $p^{\prime}$-subgroup of $G$. Since $D(G)=1$, there is a maximal subgroup $H$ which does not contain $M$. Then $M H=G$, and, since $M$ is abelian, $M \cap H$ is normal in $M H$. Hence $M \cap H=1$, and so $H$ is a complement to $M$ in $G$. Then $|B|=|G / M|$ which implies that $H$ contains a Hall $p^{\prime}$-subgroup of $G$. Replacing $H$ by a conjugate if necessary, we may assume that $H$ contains $J K$.

Since $C_{H}(M)$ is normal in $M H=G$ and $M \cap H=1$, we must have $C_{H}(M)=1$. Hence $C_{G}(M)=M C_{H}(M)=M$. Next, $M \cap Z(E(G))$ is a nonidentity normal subgroup of $G$. The minimality of $M$ implies that $M \subseteq Z(F(G))$. Then $F(G) \subseteq C_{G}(M)=M$. Hence $M=F(G)$. If $G=M$, then $G$ can have no proper non-identity subgroup which would imply that $|G|=p$. Since $A$ and $B$ are both proper and $G=A B$, this is impossible. Thus $G \neq M$.

Since $M \subseteq P Q$ and $[P Q, J K]=1, J \cap K \subseteq C_{G}(M)=M$. Therefore $J \cap K=1$. Now let $R=O_{p^{\prime}}(H) . H$ is isomorphic to $G / M$ and $M=o_{p}(G)$. Hence $o_{p}(H)=1$. It follows from this that $C_{H}(R)=Z(R)$. Now $C_{M}(R)$ is normal in $M H=G$ and $M$ is a minimal normal subgroup of $G$. Thus $C_{M}(R)$ is either 1 or $M$. But $C_{M}(R)=M$ would imply that $R \subseteq C_{G}(M)=M$, an impossibility. Thus $C_{M}(R)=1$. Since $C_{G}(R) \subseteq C_{H}(R) M=Z(R) M$ and $C_{M}(R)=1$, we must have $C_{G}(R)=Z(R)$. But $R \subseteq J K$ and $[J K, P \cap Q]=1$. Hence $P \cap Q=1$, which implies that $A \cap B=1$. Therefore $|G|=|A||B|$.

Now let $A_{1}=A M \cap B$ and $B_{1}=B M \cap H$. Since $A M=M(A M \cap H)$ and $A_{1} \cap M=H \cap M=1, A_{1}$ is isomorphic to $A M / M$ which is isomorphic to the nilpotent group $A /(A M)$. Similarly, $B_{1}$ is isomorphic to $B /(B N M)$. Clearly $J$ and $K$ are Hall $p$-subgroups of $A_{1}$ and $B_{1}$, respectively. Thus if $L$ is a p-subgroup of $A_{1} \cap B_{1}$, then $[R, L] \subseteq[J K, L]=1$. 
Since $C_{G}(R)$ is a $p^{\prime}$-group, it follows that $A_{1} \cap B_{1}$ is a $p^{\prime}$-group. Then $A_{1} \cap B_{1} \subseteq J \cap K=1$. Hence

$$
\begin{aligned}
\left|A_{1} B_{1}\right|=\left|A_{1}\right|\left|B_{1}\right| & =|A||B| /(|A \cap M||B \cap M|) \\
& =|G| /|(A \cap M)(B \cap M)| \geq|G| /|M|=|H| .
\end{aligned}
$$

Since $A_{1} B_{1} \subseteq H$, this implies that $A_{1} B_{1}=H$ and $M=(A \cap M)(B \cap M)$.

Now $A \cap M \subseteq C_{M}(J)$. Therefore $C_{M}(J)=\langle A \cap M)\left(B \cap M \cap C_{M}(J)\right)$. But $C_{M}(J) \cap B \cap M \subseteq C_{M}(J K) \subseteq C_{M}(R)=1$. Thus $C_{M}(J)=A \cap M$. Similarly $C_{M}(K)=B \cap M$.

Suppose now that $[J, K]=1$. Then $C_{M}(J)$ and $C_{M}(K)$ are both normalized by $J K$. Since $C_{M}(J) \cap C_{M}(K)=1$, $C_{M}(J)=\left[C_{M}(J), K\right] \subseteq[M, K]$. Since $M=[M, K] \times C_{M}(K)$ and $M=C_{M}(J) \times C_{M}(K)$, we must have $[M, K]=C_{M}(J)$. Similarly . $[M, J]=C_{M}(K)$. Since $P$ normalizes $J$ and $Q$ normalizes $K$, it follows that $P Q$ normalizes both $C_{M}(J)$ and $C_{M}(K)$. Thus $C_{M}(J)$ and $C_{M}(K)$ are normal in $(J P)(K Q)=A B=G$. Due to the minimality of $M$, one of $C_{M}(J)$ and $C_{M}(K)$ is 1 . Assume, say, that $C_{M}(J)=1$. Then $C_{M}(K)=M$. Hence $K \subseteq C_{G}(M)=M$ and so $K=1$. Then $P \subseteq C_{G}(J)=C_{G}(J K) \subseteq C_{G}(R)=Z(R)$ which implies that $P=1$. It now follows that $A$ is a Hall $p^{\prime}$-subgroup and $B$ is a sylow p-subgroup.

We now assume that $[J, K] \neq I$ and derive a contradiction. Let $T=[J, K]$. Since $T$ is a $p^{\prime}$-group; $[M, T] \neq 1$. From Maschke's Theorem, there is a subgroup $N$ in $M$ such that $N$ is a minimal normal subgroup in $M J K$ and $[N, T] \neq 1$. If $x \in N$, then $x=y z$ for some $y \in C_{M}(J)$ and $z \in C_{M}(K)$. Then $N \supseteq[x, J]=[z, J]$. It follows from this that $N z \in C_{M / N}(J K)=C_{M}(J K) N / N=N / N$. Hence $z \in C_{N}(K)$. Similarly, $y \in C_{N}(J)$. It now follows that $N=C_{N}(J) \times C_{N}(K)$.

Suppose that $C_{N}(J)=1$. Then $[N, K]=1$. In that case 
$[N, K, J]=1$ and $[N, J, K]=[N, K]=1$. The three subgroups lemma yields $[T, N]=1$. Hence $C_{N}(J) \neq 1$. Similarly $C_{N}(K) \neq 1$. Then both $[N, J]$ and $[N, K]$ are proper subgroups of $N$.

Let $S$ be a maximal subgroup of $N$ containing $[N, J]$. Then $J$ normalizes $S$ and so $\left(S \cap C_{N}(K)\right)^{M K J}=\left(S \cap C_{N}(K)\right)^{J} \subseteq S$. Due to the minimality of $N$, we must have $S \cap C_{N}(K)=1$. Since $|N / S|=p$, we must have $\left|C_{N}(K)\right|=p$. Similarly $\left|C_{N}(J)\right|=p$ and so $|N|=p^{2}$.

Then $|[N, J]|=p$. Since the automorphism group of a group of order $p$ is abelian, $J / C_{J}(N)$ must be abelian. Similarly $K / C_{K}(N)$ is abelian. Now let $U=J K / C_{J K}(N), J_{1}=J C_{J K}(N) / C_{J K}(N)$, and $K_{1}=K C_{J K}(N) / C_{J K}(N)$. Then $J_{1}$ and $K_{1}$ are abelian and $U=J_{1} K_{1}$. A theorem of 1 tô [4] implies that there is a non-identity normal subgroup of $U$ contained in either $J_{1}$ or $K_{1}$. Assume then that $L$ is a non-identity normal subgroup of $U$ and $L \subseteq J_{1}$. Then $C_{N}(L)$ is normal in MJK and $C_{N}(L) \neq N$. The minimality of $N$ implies that $C_{N}(L)=1$ which contradicts $C_{N}(L) \supseteq C_{N}\left(J_{1}\right)=C_{N}(J) \neq 1$. This contradiction finishes the proof of the theorem.

\section{The main theorems}

For the rest of the paper, we assume that $A$ and $B$ are nilpotent subgroups of the group $G$ such that $G=A B$.

THEOREM 2. $a(G / D(G)) \leq c(A)+c(B)$.

Proof. Let $G$ be a minimal counter-example and let $n=c(A)+c(B)$. If $N$ is a non-identity normal subgroup of $G$, then aue to the minimality of $G$ we must have $d((G / N) / D(G / N)) \leq n$. Hence $G / N \in F_{n}$ but $G \mid F_{n}$. Since the theorem is certainly true if $G$ is nilpotent, $A$ and $B$ must be proper. Applying Lemma 1 and Theorem 1, we find that $(|A|,|B|)=1$ and $D(G)=1$. The theorem now follows from [3, Theorem 1.2.4].

THEOREM 3. Asswme that the Sy low 2-subgroups of both $A$ and $B$ 
have class at most 2 . Then

$$
d(G / D(G)) \leq d(A) d(B)+1 .
$$

Proof. Let $G$ be a minimal counter-example and let $n=d(A) d(B)+1$. Then $F_{n}$ does not contain $G$ but does contain every proper homomorphic image of $G$. By Lemma $I, D(G)=1$ and $G$ has exactly one minimal normal subgroup $M, A$ and $B$ must be proper and so the hypothesis of Theorem 1 is satisfied. Thus, without loss of generality, we may assume that $A$ is a Sylow p-subgroup of $G$, that $M=O_{p}(G)=F(G)$, and that $B$ is a Hall $p^{\prime}$-subgroup of $G$. Lemma 2 implies that $d(A / M)=d(A)-1$. Then

$$
d((G / M) / D(G / M)) \leq(d(A)-1) d(B)+1 .
$$

Hence, if $m=n-d(B)$ and $H=G / M$, then $H \in F_{m}$. As pointed out earlier, $H \in F_{m}$ if, and only if, $H^{(m-1)}$ is nilpotent. Thus $d(H / F(H)) \leq m-1$. Since $o_{p}(H)=1, d(F(H)) \leq d(B)$. Thus $d(H) \leq m-1+d(B)$. This implies that $d(G) \leq d(H)+1 \leq n$.

THEOREM 4. Asswme that the Sylow 2-subgroups of both $A$ and $B$ have class at most 3 . Then $Z(G) \leq d(A)+d(B)$.

Proof. Let $G$ be a minimal counter-example and let $r=d(A)+d(B)$. Then $N_{r}$ contains every proper homomorphic image of $G$ but does not contain $G$. Therefore, as in the proof of Theorem 3, we may assume that $F(G)=O_{p}(G)$, that $A$ is a sylow p-subgroup of $G$, and that $B$ is a Hall $p^{\prime}$-subgroup of $G$. Let $H=G / F(G)$. Then $Z(G)=Z(H)+1 \leq d(B)+d(A / F(G))+1$. Since $G$ is a counter-example, we must have $d(A / F(G))=d(A)$. It follows from Lemma 2 that $p=2$ and $c(A)=3$. Then Lemma 3 implies that $z_{2}(G) \leq 2$. From this, we obtain $Z(G) \leq 4$. Since $Z(G)>d(A)+d(B)$, we must have $d(B)=1$. Since $F(H)$ is a $2^{\prime}$-group and $C_{H}(F(H)) \subseteq E(H)$, it follows that $H / F(H)$ is a 2-group. Therefore $Z(H) \leq 2$. Then $Z(G) \leq 3=d(A)+d(B)$ and the theorem is proved. 


\section{References}

[1] Wolfgang Gaschütz, "Zur Theorie der endlichen auflösbaren Gruppen", Math. Z. 80 (2963), 300-305.

[2] Fletcher Gross, "The 2-length of groups whose Sylow 2-groups are of exponent 4 ", J. Algebra 2 (1965), 312-314.

[3] P. Hall and Graham HIgman, "On the p-length of p-soluble groups and reduction theorems for Burnside's problem", Proc. London Math. Soc. (3) 6 (1956), 1-42.

[4] Noboru 1tô, "Über das Produkt von zwei abelschen Gruppen", Math. 2. 62 (1955), 400-401.

[5] Otto H. Kegel, "Produkte nilpotenter Gruppen", Arch. Math. 12 (1961), 90-93.

[6] Helmut Wlelandt, "Über das Produkt paarweise vertauschbarer nilpotenter Gruppen", Math. 2. 55 (1951), 1-7.

Department of Mathematics,

The University of Utah,

Salt Lake City,

Utah,

USA. 